\title{
Cyanidin Curtails Renal Cell Carcinoma Tumorigenesis
}

\author{
Xiaobing Liu ${ }^{\mathrm{a}}$ Dangling Zhang ${ }^{\mathrm{a}}$ Yaxing Hao $\mathrm{Ha}^{\mathrm{b}}$ Qian Liu ${ }^{\mathrm{a}}$ Yuqi Wu \\ Xin Liu ${ }^{a}$ Jing Luo ${ }^{a}$ Tao Zhou ${ }^{a}$ Bishao Sun ${ }^{a}$ Xing Luo ${ }^{a}$ Jie Xu \\ Qingqing Wang ${ }^{\mathrm{a}}$ Zhenxing Yang ${ }^{\mathrm{a}}$ Longkun Lia
}

${ }^{a}$ Department of Urology, Xinqiao Hospital, Third Military Medical University, Chongqing, ' ${ }^{b}$ Institute of Immunology, Third Military Medical University, Chongqing, China

\section{Key Words}

Renal cell carcinoma $\cdot$ Cyanidin $•$ Apoptosis $•$ Migration $・$ Invasion

\begin{abstract}
:
Background/Aims: Cyanidin is an anthocyanin found in many foods. Although its variable antioxidant levels are well-documented, little is known about its effects on renal cell carcinoma (RCC) tumorigenesis. This study, therefore, investigated the effects of cyanidin on the proliferation, migration, and invasion of renal cell carcinoma lines and demonstrated, for the first time, significant inhibitory effects of cyanidin on RCC tumorigenesis. Methods: RCC cells were treated with different doses of cyanidin and the effects were tested by Cell Counting Kit8 reagent, clone formation assay, transwell assay, and flow cytometry. Moreover, the cyanidinmediated mechanism that curtailed tumorigenesis was analyzed by RNA sequencing (RNAseq). Sequencing data from The Cancer Genome Atlas (TCGA) were used to compare the expression of both early growth response protein 1 (EGR1) and selenoprotein W (SEPW1) in RCC and tumor-free adjacent normal tissue samples. Real-time PCR (RT-PCR) and/or western blot were used to assess the expression of E-cadherin, cleaved-caspase3, Bcl2, p62, and ATG4. Results: We found significantly greater induction of cell-cycle arrest, apoptosis, and suppression of RCC cell invasion and migration at concentrations of $25 \mu \mathrm{M}$ and $100 \mu \mathrm{M}$ than at a concentration of $50 \mu \mathrm{M}$. It was also discovered, first through RNA-seq then confirmed by RT-PCR, that cyanidin $(100 \mu \mathrm{M})$ inhibited RCC carcinogenesis through EGR1 and SEPW1. TCGA data indicated that the expression level of EGR1 was lower and that of SEPW1 was higher in RCC tumor tissue than in normal tissues. Moreover, western blot and/or RT-PCR indicated that cleaved-caspase 3 was enhanced and E-cadherin was inhibited by cyanidin treatment. Furthermore, western blot and RT-PCR also showed regulation of p62 and ATG4, which are associated with autophagy. Cyanidin in vivo significantly inhibited the growth of xenografts in nude mice. Conclusions: The results of this study showed the therapeutic potential of cyanidin for the treatment of RCC and the prevention of recurrence and metastasis.
\end{abstract}

(C) 2018 The Author(s)

Published by S. Karger AG, Basel

Longkun Li, MD, PhD

KARGER
Department of Urology, Xinqiao Hospital, Third Military Medical University Chongqing, 400037 (China)

Tel. +862368755623; E-Mail lilongk@hotmail.com, liuxiaobing10@tmmu.edu.cn 


\section{Introduction}

Renal cell carcinoma (RCC) accounts for $2-3 \%$ of all malignant diseases in adults, and is the most common type of kidney cancer. Currently, surgical resection of the kidneys remains the most effective treatment for RCC because almost all RCC pathological types are resistant to chemotherapeutic and radiation therapies. However, roughly one-third of patients with RCC are diagnosed during the later phases when the cancer has progressed to the point that significant opportunities for surgical treatment have already been missed. Although immunotherapy is currently a key treatment for the late phase of the disease, the response rate is less than 20\% [1], and while the responses of metastatic RCC to sorafenib and sunitinib are better $[2,3]$, the overall survival rate remains poor. Therefore, alternative anti-tumor agents and methods that are highly efficient are strongly needed. The identification of novel therapeutic molecules for RCC will help to provide an understanding of the pathogenesis that leads to tumorigenesis and improve outcomes for patients.

Recently, numerous natural products have served as important sources for the development of potent anti-tumor drugs [4, 5]. Flavonoids have unique and varied chemical structures and include a large variety of polyphenolic compounds that occur naturally in plant foods [6]. Extensive studies have shown that the consumption of dietary flavonoids affords significant anti-tumor effects [7-10]. Moreover, there have been multiple studies performed at the cellular level to investigate the effects of flavonoids on tumor proliferation, migration, and invasion [11-13]. This study focused on cyanidin, which belongs to a branch of the flavone compounds. Cyanidin is a well-defined anthocyanidin compound that occurs in fruits and vegetables [14] and has various biological functions such as antioxidant and antiinflammatory activities [15-17]. Cyanidin is not the most common dietary anthocyanidin, however, and it occurs naturally in its glycosylated form, namely, cyanidin-3-glucoside (C3G). After intake, C3G can be metabolized by oral epithelial cells and absorbed by the gastric epithelium $(1-10 \%)$, where it is gut-transformed and enters the bloodstream $(<1 \%)$ and urine (about $0.02 \%$ ) [18]. After oral administration, the predominant flavonoid species in the gastrointestinal (GI) mucosa and liver is C3G, whilst after IV dosing the majority of anthocyanins are C3G metabolites, which are mostly products of methylation and glucuronidation. Cyanidin is a minor metabolite in the gut [19]. The effects of anthocyanidins on chronic diseases, carcinomas, and health promotion have been intensively studied over the last few decades [14, 20-24], but the impact of cyanidin on renal cell carcinoma has not yet been revealed.

We therefore investigated the effects of cyanidin on RCC and demonstrated its role in RCC cell proliferation, invasion, apoptosis, and the cell cycle in vitro. The results of our investigations showed that cyanidin-induced cell-cycle arrest and apoptosis and the suppression of RCC cell invasion and migration were significantly greater at concentrations of $25 \mu \mathrm{M}$ and $100 \mu \mathrm{M}$ than at concentrations of $50 \mu \mathrm{M}$. Moreover, the RNA-seq results indicated that cyanidin $(100 \mu \mathrm{M})$ increased the expression of EGR1 and decreased the expression of SEPW1 in RCC cell lines compared with a control group and confirmed by RTPCR. Previous research has shown that quercetin (a flavonoid) induces apoptosis in colon carcinoma cells via EGR1 [25], and that the silencing of SEPW1 mRNA expression causes cells to accumulate in the G0/G1 phase of the cell cycle via P53 and p21 [26]. The present study demonstrated that cyanidin mediated its anticancer effects via EGR1 and SEPW1 and provided a mechanistic basis for the anticancer effects on RCC cells. Furthermore, western blot assay indicated that both the cleavage of the apoptotic mediator caspase 3 and inhibition of E-cadherin activation were enhanced, while additional western blot and RT-PCR revealed the regulation of both P62 and ATG4, which are associated with autophagy. In vivo, cyanidin $(0.5 \mathrm{mg} /$ mouse) significantly inhibited the growth of xenografts in the nude mouse model; however, the anti-proliferation activities of cyanidin and its underlying mechanisms on cancer cells remain largely unknown. If the mechanisms can be elucidated in detail, they may provide future guidance for the development of cyanidin as a potent anticancer agent.

\section{KARGER}




\section{Cellular Physiology Cell Physiol Biochem 2018;46:2517-2531 \\ \begin{tabular}{l|l|l} 
DOI: 10.1159/000489658 & O 2018 The Author(s). Published by S. Karger AG, Basel \\
www.karger.com/cpb
\end{tabular}}

Liu et al.: Cyanidin Curtails RCC Tumorigenesis

\section{Materials and Methods}

\section{Protocol approval}

All experiments were carried out in strict accordance with the regulations and guidelines for experimental research issued by the Third Military Medical University, as well as the protocol of the Institutional Animal Care and Use Committee of the Third Military Medical University.

\section{Cell lines, reagents, and antibodies}

The 786-0 and ACHN cell lines were obtained from the American Type Culture Collection (Rockville, MD, USA). Line 786-0 was grown in RPMI-1640 supplemented with 10\% fetal bovine serum (FBS) in a humidified chamber with $5 \% \mathrm{CO}_{2}$ at $37^{\circ} \mathrm{C}$; ACHN cells were grown in MEM supplemented with $10 \%$ FBS. FBS was purchased from Gibco (Life Technologies, CA, USA). The penicillin-streptomycin solution was Hyclone (Thermo Fisher Scientific, Massachusetts, USA). The cell strain was purchased from Corning (Corning, NY, USA). Cyanidin was purchased from Sigma-Aldrich Corporation (St. Louis, MO, USA). Antibodies against MMP-9, Cyclin D1, E-cadherin, HIF2A, Bcl2, cleaved caspase-3, LC3, and p62 were purchased from Cell Signaling Technology (Danvers, MA).

\section{Cell proliferation assay}

The 786-0 and ACHN cells were seeded $\left(2 \times 10^{3}\right.$ per well $)$ into 96-well plates and left to adhere overnight. The medium was removed the following day and fresh medium, either alone or with cyanidin $(0$, $0.5,1,2,5,10,25,50,75$, or $100 \mu \mathrm{M}$ ) was added. The cultures were incubated for either $24 \mathrm{~h}, 48 \mathrm{~h}$, or 72 h. Floating cells were then removed and $100 \mu \mathrm{L}$ of fresh medium containing 1/10 (v/v) Cell Counting Kit-8 reagent (CCK-8, Dojindo Laboratories, Kumamoto, Japan) was added to each well before incubation for a further $4 \mathrm{~h}$. After incubation, the absorbance was measured using a 96-well plate reader at $450 \mathrm{~nm}$. Wells containing CCK-8 reagent without cells were used as blank controls. Cell proliferation was assessed by the absorbance values according to the manufacturer's protocol.

\section{Clone formation assay}

Cells were seeded at a density of 200 cells on 6-well plates and maintained in a complete medium containing 10\% FBS with different concentrations of cyanidin for 12 days. Colonies were fixed with methanol and stained with $0.1 \%$ crystal violet (Beyotime Institute of Biotechnology, Shanghai, China). Visible colonies were photographed and counted manually.

\section{Apoptosis assay}

The cyanidin-induced apoptosis of 786-0 and ACHN cells was detected by flow cytometry using an Annexin V-FITC Apoptosis Detection Kit (KGA, KeyGEN, Biotech, City, China) according to the manufacturer's instructions. The rate of apoptosis was analyzed using FACSCalibur Flow Cytometer (Becton Dickinson and Beckman-Coulter, San Jose, CA, USA) at $488 \mathrm{~nm}$, and the data were analyzed by flow cytometry (MoFlo, Beckman, CA, USA).

\section{Cell cycle detection assay}

On reaching $60-80 \%$ confluence, cells were treated with different amounts of cyanidin $(0,25,50$, or $100 \mu \mathrm{M}$ ) for $48 \mathrm{~h}$. The cells were collected and washed twice with phosphate-buffered saline (PBS), fixed with $70 \%$ ethanol for $1 \mathrm{~h}$ at $4{ }^{\circ} \mathrm{C}$, washed again with PBS, and re-suspended with propidium iodide (PI) solution $(0.05 \mathrm{mg} / \mathrm{mL})$ containing RNase. Next, they were incubated at room temperature in the dark for 30 min. The DNA content was analyzed using a flow cytometer (MoFlo, Beckman); the populations of cells in each phase of the cell-cycle were determined using Flow Jo 10.6 for Windows (Tree Star, Ashland, OR, USA).

\section{Wound healing assay}

A wound healing assay was used to assess the capacity of the cells to migrate and invade. When cells reached $90-95 \%$ confluence, a wound was generated by scratching the surface of the plates with a pipette tip. The floating cells were removed, and the adherent cells were incubated with various concentrations of cyanidin $(0,25,50$, or $100 \mu \mathrm{M})$ for $24 \mathrm{~h}$ until the wound healed. The gap distances at different time points were normalized to the distance immediately after the scratch was made. 


\section{Transwell cell migration and invasion assay}

Transwell (Millipore, Billerica, MA, USA) assays were used to measure migration. A total of $4 \times 10^{4} 786$ 0 and ACHN cells were seeded into the upper chamber in serum-free media with different concentrations of cyanidin $(0,25,50$, or $100 \mu \mathrm{M})$. The medium was further supplemented with $10 \%$ serum, which was added to the lower chamber (it contained the appropriate concentration of cyanidin). After $24 \mathrm{~h}$ of incubation the cells were fixed and stained using $0.05 \%$ crystal violet. The numbers of cells that migrated from the upper to the lower chamber were counted in five random fields of view per experiment and the assays were performed in triplicate.

For the invasion assay, a mixture of Matrigel (Millipore) and serum-free cell culture medium (1:10) was added to the upper chamber of the 24 -well transwell plates, incubated at $37^{\circ} \mathrm{C}$ for $4 \mathrm{~h}$ for gelling, and kept at $4{ }^{\circ} \mathrm{C}$ for $24 \mathrm{~h}$. RPMI 1640 culture medium plus $20 \% \mathrm{FBS}(600 \mu \mathrm{L} /$ well $)$ was added to the bottom well. Cells $\left(4 \times 10^{4}\right.$ in $\left.100 \mu \mathrm{L}\right)$ were subsequently seeded in the upper chamber with an $8-\mu \mathrm{m}$ pore membrane and incubated at different concentrations of cyanidin $(0,25,50$, or $100 \mu \mathrm{M})$ for $24 \mathrm{~h}$. The cells in the membrane were fixed with $4 \%$ PFA for $10 \mathrm{~min}$, stained with $0.1 \%$ crystal violet for $5 \mathrm{~min}$, and counted.

\section{$R T-P C R$}

The total RNA was isolated using a TRIzol Reagent (Life Technologies, CA, USA). Single-strand cDNA was prepared from $1 \mu \mathrm{g}$ of total RNA using reverse transcriptase with oligo(dT) primer according the manufacturer's instructions (Takara Bio, Japan). Each cDNA sample $(2 \mu \mathrm{L})$ was subjected to RT-PCR amplification using specific primers for Bcl2, Bim, Bix, MM9, CD47, ATG4, and LC3B, as detailed in Table 1. The data were collected and analyzed using the $2^{-\Delta \Lambda} \mathrm{Ct}$ method. The values were normalized to those of GAPDH and then compared with the experimental controls.

\section{Detection of reactive oxygen species}

The dichloro-dihydro-fluorescein diacetate (DCFH-DA; Beyotime) method was used to measure intracellular reactive oxygen species (ROS) levels. The cells were harvested following treatment with cyanidin and washed once with ice-cold PBS before treatment with DCFH-DA (at a final concentration of $10 \mathrm{~mol} / \mathrm{L}$ in a serum-free medium). After incubation at $37^{\circ} \mathrm{C}$ for $20 \mathrm{~min}$ in the dark, the cells were washed twice with PBS. The intracellular accumulation of ROS was measured by flow cytometry and the median fluorescence intensity values were calculated. All experiments were performed in triplicate.

\section{Western blot}

Cells were lysed by lysis buffer containing $10 \mathrm{mM}$ Tris, pH 7.2, $150 \mathrm{mM} \mathrm{NaCl}, 5 \mathrm{mM}$ ethylenediaminetetraacetic acid, $0.1 \%$ sodium dodecyl sulfate (SDS), $1 \%$ Triton $\mathrm{X}-100$, and $1 \%$ deoxycholic acid. The supernatants were collected after centrifugation at $12000 \times \mathrm{g}$ at $4{ }^{\circ} \mathrm{C}$ for 5 min. Protein concentration was determined using a BCA protein kit (Beyotime) and whole lysates were mixed with $4 \times$ SDS loading buffer (125 mmol/Tris-HCl, 4\% SDS, $20 \%$ glycerol, $100 \mathrm{mmol} / \mathrm{L}$ DTT, and $0.2 \%$ bromophenol blue) at a ratio of $1: 3$. Protein samples were heated at 100 ${ }^{\circ} \mathrm{C}$ for $10 \mathrm{~min}$. For the western blot, $30 \mathrm{~g}$ of protein samples

Table 1. Nucleotide sequences of primers used for RT-PCR reactions

\begin{tabular}{lll}
\hline Gene & Forward & Reverse \\
\hline Atg3 & 5'-ATGGGAGTTGGCGAAGGCAAGT-3' & 5'-AGCTCCACGTATCGAAGACAGC -3' \\
Atg4 & 5'- - TATTGGTGGCAAACCTAAACAGTC -3' & 5'- 'TTGGGAGAAGGGCAGTGGAATGA -3' \\
Atg5 & 5'-GCAGATGGACAGTTGCACACA -3' & 5'-GAGGTGTTTCCAACATTGGCTCA-3' \\
Atg7 & 5'-CGTTGCCCACAGCATCATCTTC-3' & 5'-CACTGAGGTTCACCATCCTTGG-3' \\
Atg9 & 5'-GCTTCCTCAAGGAGCAGGTTCA -3' & 5'- CCACATTTGCGATAAGGCTCAGG-3, \\
Atg10 & 5'-CTACGGAAGAGAACCAGGAGCT -3' & 5'-CTGGTAGAGGTTCCTTTGCTGC-3, \\
Atg12 & 5'-GGGAAGGACTTACGGATGTCTC -3' & 5'-AGGAGTGTCTCCCACAGCCTTT-3' \\
LC3B & 5'-CTGAGATTGGTGTGGAGACG-3' -3, & 5'-CGGTGATAATAGAACGATACAAGG -3' \\
Bak1 & GACGCCCATTCCTGGAAACT & CTTGCCCGAAGCCATTTTT \\
Bax1 & TGCTTCAGGGTTTCATCCAG & GGCGGCAATCATCCTCTG \\
Bim1 & ACTCTCGGACTGAGAAACGC & CTTCACCTCCGTGATTGCCT \\
Bcl2 & TGTGGAGAGCGTCAACCGGGAG & GCAAGCTCCCACCAGGGCCAAA \\
HIF1A & 5'-TCCAAGAAGCCCTAACGTGT-3' & 5'-TGATCGTCTGGCTGCTGTAA-3' \\
HIF2A & 5'-TGGAGACACTGGCACCCTAT-3' & 5'-GCTGCTGCTGAAACTTGTCC-3' \\
CD47 & 5'-TGCTATACTCCTGTTCTGGGGA-3' & 5'-TCTCATCCATACCACCGGATCT-3' \\
MMP9 & 5'-CACCTCGAACTTTGACAGCG-3' & 5'-CCCTCAGTGAAGCGGTACAT-3' \\
CXCR4 & 5'-GGTGGTCTATGTTGGCGTCT-3' & 5'-GTCATTGGGGTAGAAGCGGT-3' \\
SEPW1 & 5'GTCGTTTATTGTGGCGCTTGAG 3' & 5'-CCACGTAGCCATCGCCTTTCTTC-3' \\
ERG1 & 5'-CCCGGGCCACCACCTCATAC-3' & 5'-ACGGGGAGGGGAAGCCACTG-3' \\
GAPDH & 5'-GAAGGTGAAGGTCGGAGT-3' & 5'-GAAGATGGTGATGGGATTTC-3' \\
\hline
\end{tabular}


Liu et al.: Cyanidin Curtails RCC Tumorigenesis

were subjected to SDS-polyacrylamide gel electrophoresis, transferred onto a polyvinylidene fluoride membrane, and incubated first with rabbit antibodies against E-cadherin, Bcl2, cleaved caspase-3, HIF2A, p62, LC3, and actin (all from Cell Signaling Technology) overnight at $4{ }^{\circ} \mathrm{C}$, followed by another $2 \mathrm{~h}$ incubation with a secondary antibody (1:5000). GAPDH served as a loading control. The secondary antibodies were horseradish peroxidase (HRP)-conjugated anti-rabbit (Jackson ImmunoResearch Labs, West Grove, PA, USA). Densitometric analysis of the western blots was performed with NIH Image J software (Bethesda, MD, USA). The protein levels were first normalized to actin and subsequently to the experimental controls.

\section{RNA-seq and analysis}

Total RNA was prepared from 786-0 cells via RNeasy Mini Kit (Qiagen), according to the manufacturer's instructions. Library preparation and sequencing on an Illumina HiSeq2000 instrument were performed by Genewiz Inc. (New Jersey, USA). Target genes were verified by synthesizing cDNA using a qScript cDNA Synthesis Kit (Quantabio, Maryland, USA) according to the manufacturer's instructions. The RT-PCR mixtures contained 2x QuantiTect SYBR Green PCR Master Mix (Qiagen), 10x QuantiTect primer assay mix, and synthesized cDNA. Amplification was performed in triplicate for each sample on a RealPlex MasterCycler (Eppendorf, Hamburg, Germany). Relative expression levels were normalized to GAPDH levels. Eleven lowquality nucleotides from the 5' ends of the reads were trimmed for RNA-Seq data analysis. The resulting 40-nucleotide single-ended reads were mapped to the human genome (UCSC mm10) by using TopHat with NCBI Ref-Seq genes as the reference and up to two possible mismatches. Read duplicates were removed using the same tools to reduce possible biases caused by RT-PCR. Cufflinks was used to estimate gene expression levels (transcript counts). DESeq2 (27) was used to test whether the genes were differentially expressed between the wild-type and cyanidin groups. The Benjamini-Hochberg method, which controls the false discovery rate (FDR), was used to correct for multiple comparisons. Alterations of genes shown in the volcano maps were all significant (FDR, 0.1). These genes were clustered into functional groups by means of DAVID bioinformatics tools (NIH). Differential expression of genes selected from each category was verified by quantitative RT-PCR.

\section{Patient data mining from The Cancer Genome Atlas}

In this study, we collected sequencing data of RCC from The Cancer Genome Atlas (TCGA) dataset (https://tcga-data.nci.nih.gov and https://genome-cancer.ucsc.edu) containing 523 RCC tissues and 100 tumor-free adjacent normal tissues. The TCGA sequencing data was used to obtain the expression of both EGR1 and SEPW1 in RCC and tumor-free adjacent normal tissue samples. The expression levels of EGR1 and SEPW1 were analyzed using the TCGA portal. Overall survival rates of patients with high and low levels of EGR1 and SEPW1 were derived using the Kaplan-Meier survival plot.

\section{Xenograft assays in nude mice}

All animal handling and experimental protocols were carried out in accordance with the Guide for the Care and Use of Laboratory Animals of the Third Military Medical University, China (approval no. SYXK20070002). All efforts were made to minimize animal suffering and to reduce the number of animals used. Four-week old male BALB/c nude mice $(n=10)$ were used in this study and maintained at room temperature $\left(25 \pm 2{ }^{\circ} \mathrm{C}\right)$ under a standard $12 \mathrm{~h} / 12 \mathrm{~h}$ light-dark cycle in a specific pathogen free experimental animal room and fed a sterilized diet. ACHN cells were re-suspended to $2 \times 106$ cells/100 $\mu \mathrm{L}$ in PBS and implanted subcutaneously into the flank region of 6- to 7-week-old male nude mice weighing 18-25 g. When tumors reached $40-50 \mathrm{~mm}^{3}$ in volume, the animals were randomly assigned to two groups $(\mathrm{n}=5)$, receiving either intraperitoneal (IP) injections of $100 \mu \mathrm{L}$ PBS twice a week or IP injections of cyanidin (6 mg/Kg in 100 $\mu \mathrm{L}$ PBS) twice a week. Tumor volumes were measured twice a week and once the control tumors reached $1,000 \mathrm{~mm}^{3}$ the animals were humanely euthanized in accordance with ethical study requirements. At the end of the study, all animals were sacrificed with an overdose of $\mathrm{CO}_{2}$ and the tumor tissues were extracted for immunostaining and weighing.

\section{Immunohistochemistry}

Tumors excised from the nude mice were fixed in 4\% paraformaldehyde, dehydrated through a graded series of ethanol solutions, embedded in paraffin, cut into 4-mm sections, and stained with hematoxylin and eosin (H\&E). For immunohistochemistry (IHC), the sections were incubated with a primary antibody 
versus Ki-67 (Cell Signaling Technology) overnight at $4{ }^{\circ} \mathrm{C}$. The sections were subsequently incubated with biotinylated goat and rabbit antibodies IgG for $20 \mathrm{~min}$ at room temperature, and then $30 \mathrm{~min}$ with streptavidin-conjugated HRP. Diaminobenzidine- $\mathrm{H}_{2} \mathrm{O}_{2}$ was used as a substrate for peroxidase.

\section{Statistical analysis}

All data are representative of at least three experiments, which were performed in triplicate unless otherwise indicated. All statistical analyses were carried out using the GraphPad Prism 6.0 statistical software package (GraphPad Software Inc., LaJolla, CA). Data are expressed as the mean \pm standard deviation. One-way analysis of variance followed by Student-Newman-Keuls post hoc tests were used to determine the significance of the differences between the results. A p-value of $<0.05$ was regarded as significant.

\section{Results}

Cyanidin inhibits the growth and migration of RCC cells

To investigate the effect of cyanidin on the proliferation of human RCC cell lines, 786$\mathrm{O}$ and ACHN cells were treated with different concentrations of cyanidin and CCK-8 assays were performed. The corresponding IC50s for 786-0 and ACHN cell lines were $120.6 \mu \mathrm{M}$ and $96.96 \mu \mathrm{M}$, respectively (Fig. 1A). Cyanidin treatment of 786-O and ACHN cells decreased cell viability in a concentration-dependent manner, whereas cell viability was higher at 50 $\mu \mathrm{M}$ than at $25 \mu \mathrm{M}$. The effect of cyanidin was also tested on colony formation, another type of proliferation assay, in which 200 of each RCC cell type (786-0 and ACHN) were seeded into 6-well plates and cultured with cyanidin at concentrations of $0,25,50$, or $100 \mu \mathrm{M}$ for 14 days. As shown in Fig. 1B-D, cyanidin significantly inhibited colony formation of both 786-0 and ACHN cells in 25 and $100 \mu \mathrm{M}$.

The possibility of whether cyanidin would inhibit the migratory and invasive ability of RCC cells was also explored. Cyanidin concentrations of $0,25,50$, and $100 \mu \mathrm{M}$ were chosen for these experiments. In a wound healing assay, both 786$\mathrm{O}$ and ACHN cells were cocultured with different concentrations of cyanidin for $24 \mathrm{~h}$. Compared with the control group, cyanidin inhibited the migratory ability of 786-O and ACHN cells (Fig. 2A-D). Moreover, a concentration-independent inhibitory effect was observed, whereby migration decreased less at $50 \mu \mathrm{M}$ than at $25 \mu \mathrm{M}$ (Fig. 2B and 2D) Similarly, in a transwell migration and invasion assay (Fig. 2E-H), cyanidin inhibited the ability of RCC cells to migrate. These data suggest that cyanidin inhibits the proliferation and migration of RCC at a broad range of concentrations. Furthermore, cyanidin significantly curtails RCC tumorigenesis via concentration-independent effects.

\section{Cyanidin induces RCC cell apoptosis and cell cycle arrest}

The study also investigated whether the suppression of cell growth by cyanidin was due to an impact on cell apoptosis. Cell death in 786-0 and ACHN was measured by flow cytometry based

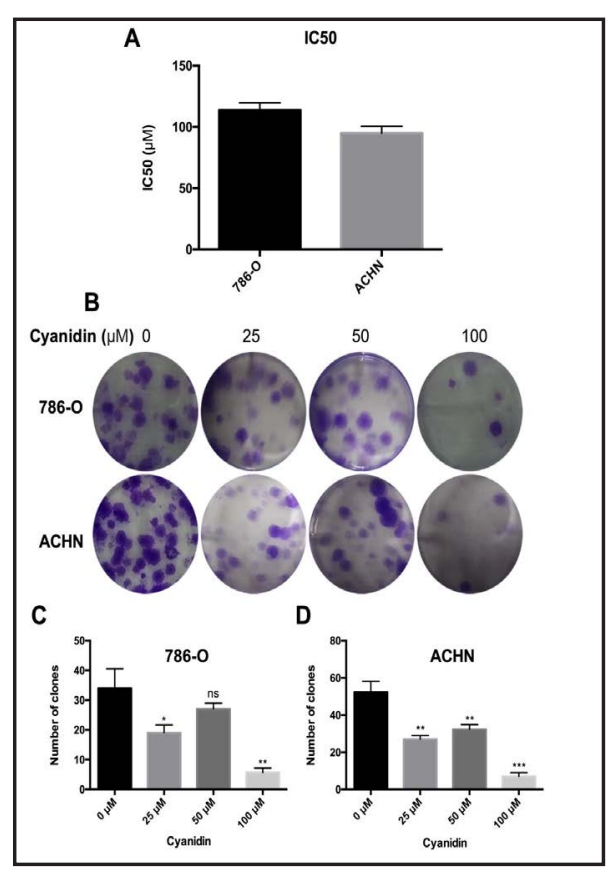

Fig. 1. Cyanidin inhibits RCC-cell proliferation and colony formation in vitro. (A) The IC50 values at the indicated concentrations of cyanidin in 786-0 and ACHN cells were detected by the CCK-8 assay and absorbance was read at $450 \mathrm{~nm}$. (B-D) A colony formation assay was performed to detect the proliferation of 786-O and ACHN cells. ${ }^{*} \mathrm{p}<0.05$ (compared with the $0 \mu \mathrm{M}$ group), ${ }^{* *} \mathrm{p}<0.01,{ }^{* * *} \mathrm{p}<0.001$. 
Fig. 2. Cyanidin induces anti-metastatic effects. Cells were treated with cyanidin $(0,25,50$, or $100 \mu \mathrm{M})$ for the indicated times (24 or $48 \mathrm{~h}$ ). (A-D) Wound healing, $(\mathrm{E}, \mathrm{F})$ transwell migration, and $(\mathrm{G}, \mathrm{H})$ invasion assays were performed. Red lines represent the borders of the wounds. The pictures shown are representative of three separate experiments. Error bars represent standard deviations. ${ }^{*} \mathrm{p}<0.05$, $* * \mathrm{p}<0.01$.

Fig. 3. Cyanidin promotes apoptosis and induces G1 arrest in RCC cells. (A-C) Flow cytometry was used to analyze apoptosis in 786-O and ACHN cells treated with cyanidin. (D-G) Flow cytometry was used to analyze cell cycle arrest in 786-0 and ACHN cells treated with cyanidin.
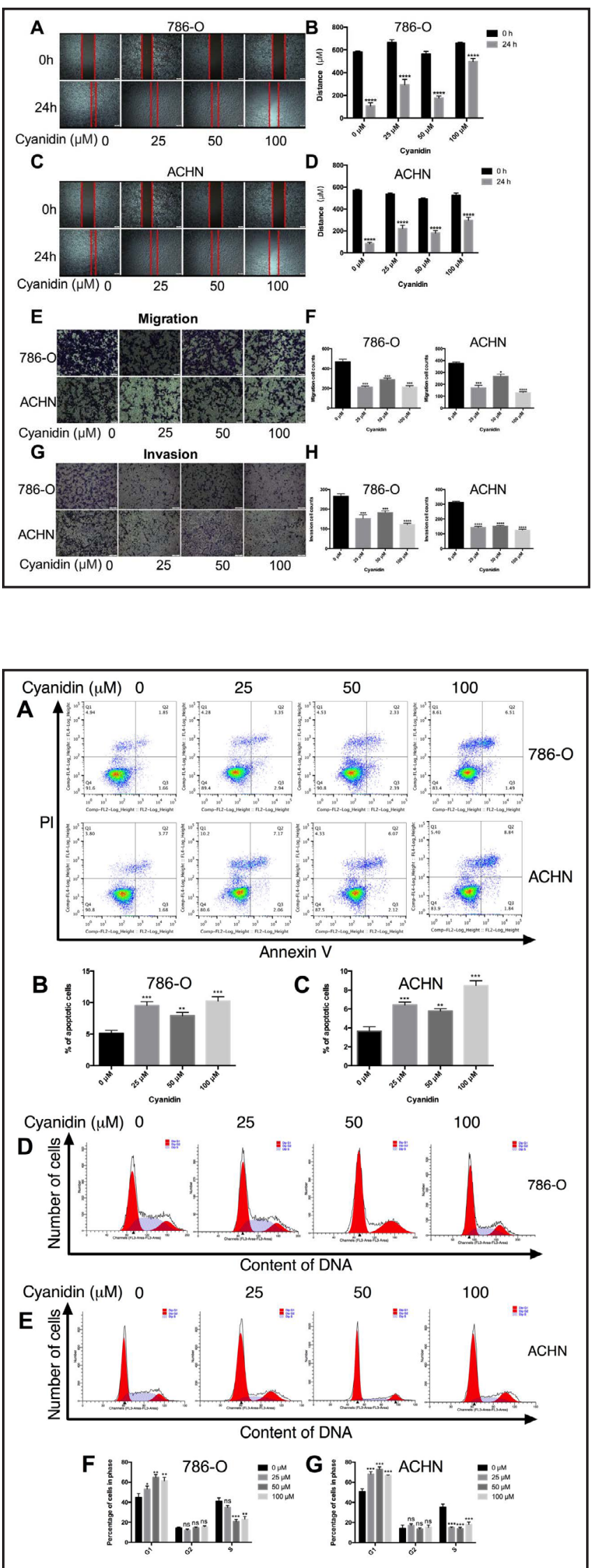
on annexin V and PI staining. The percentage of apoptotic cells gradually increased after treatment with cyanidin in a concentration-independent manner (Fig. 3A-C). Cyanidin was found to affect late apoptosis in a dose-dependent manner; however, the early apoptosis rate appeared relatively unaffected by the different concentrations.

The effect of cyanidin on cell cycle arrest was determined by flow cytometry. After cyanidin treatment for $48 \mathrm{~h}$ at $0,25,50$, and $100 \mu \mathrm{M}$, most cells arrested mainly at phase G1-M stage, independently of the concentration. Fig. 3D-G show that cyanidin induced cell cycle arrest. These results indicated that cyanidin inhibited the growth of RCC cells by inducing apoptotic death and cell cycle arrest.

Fig. 4. Cyanidin increases EGR1 expression and decreases SEPW1 expression. (A) Volcano map of RNA-seq data. (B) Gene Ontology analysis of RNA-seq data. (C) Kyoto Encyclopedia of Genes and Genomes analysis of RNA-seq data. (D-G) RT-PCR confirmed that EGR1 was upregulated and SEPW1 downregulated in 786-0 cells treated with cyanidin $(100 \mu \mathrm{M})$. (H, I) EGR1 was downregulated and SEPW1 upregulated in an RCC tumor compared with normal kidney tissue based on TCGA data. (J, K) Kaplan-Meier analysis revealed that lower expression of EGR1 mRNA correlated with a lower survival rate in RCC.
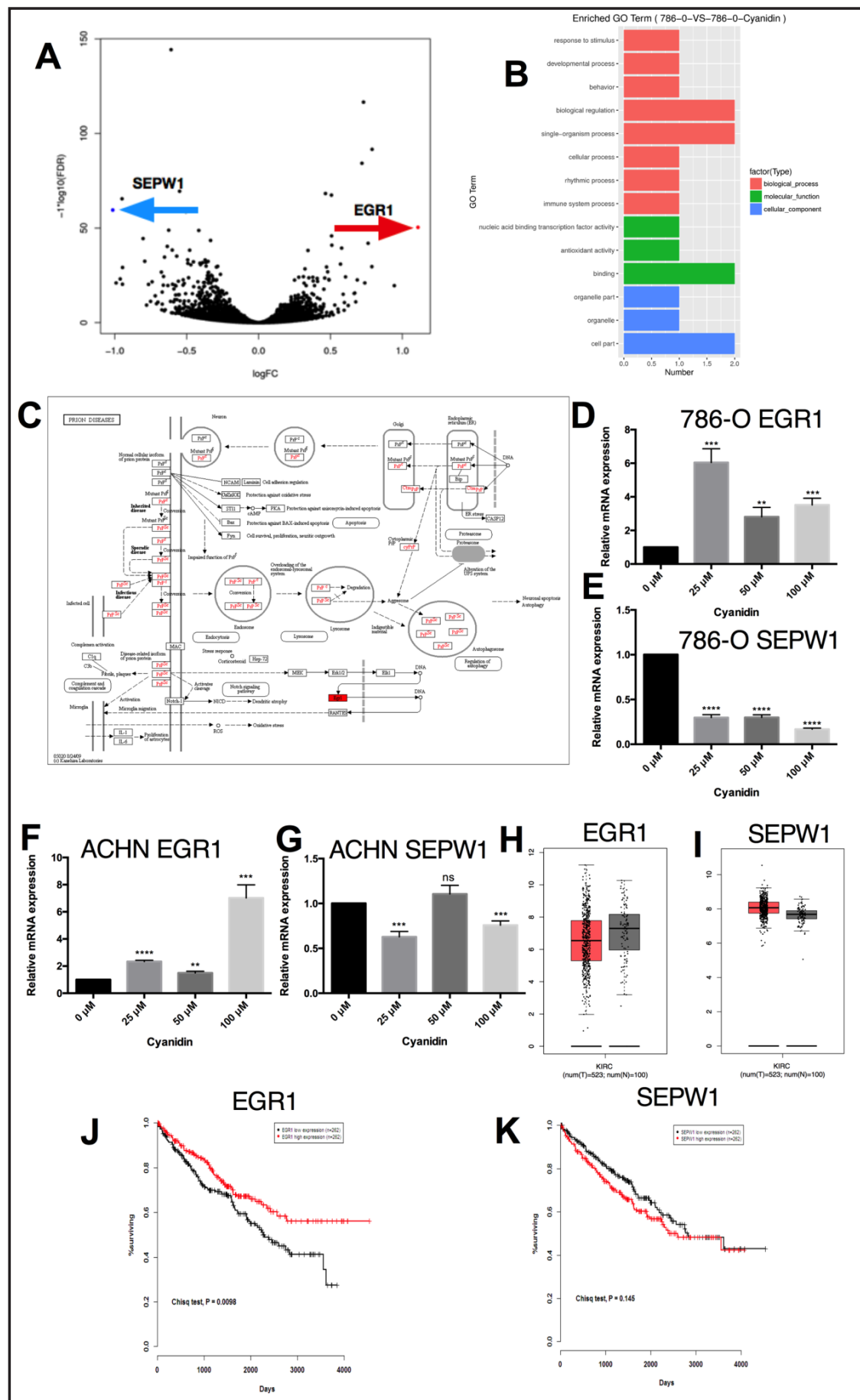
Fig. 5. Effect of cyanidin on mRNA and protein expression in RCC cells. (A, B) Bcl2 mRNA decreased when cells were co-cultured with cyanidin. (C, D) HIF2A mRNA decreased when cells were co-cultured with cyanidin. (E) E-caherin, HIF2A, Bcl2, and cleaved-caspase 3 proteins were regulated when cells were cocultured with cyanidin. $\quad{ }^{*} \mathrm{p}<0.05 \quad$ (compared with the $0 \mu \mathrm{M}$ group), ${ }^{* *} \mathrm{p}<0.01,{ }^{* * *} \mathrm{p}<0.001$.

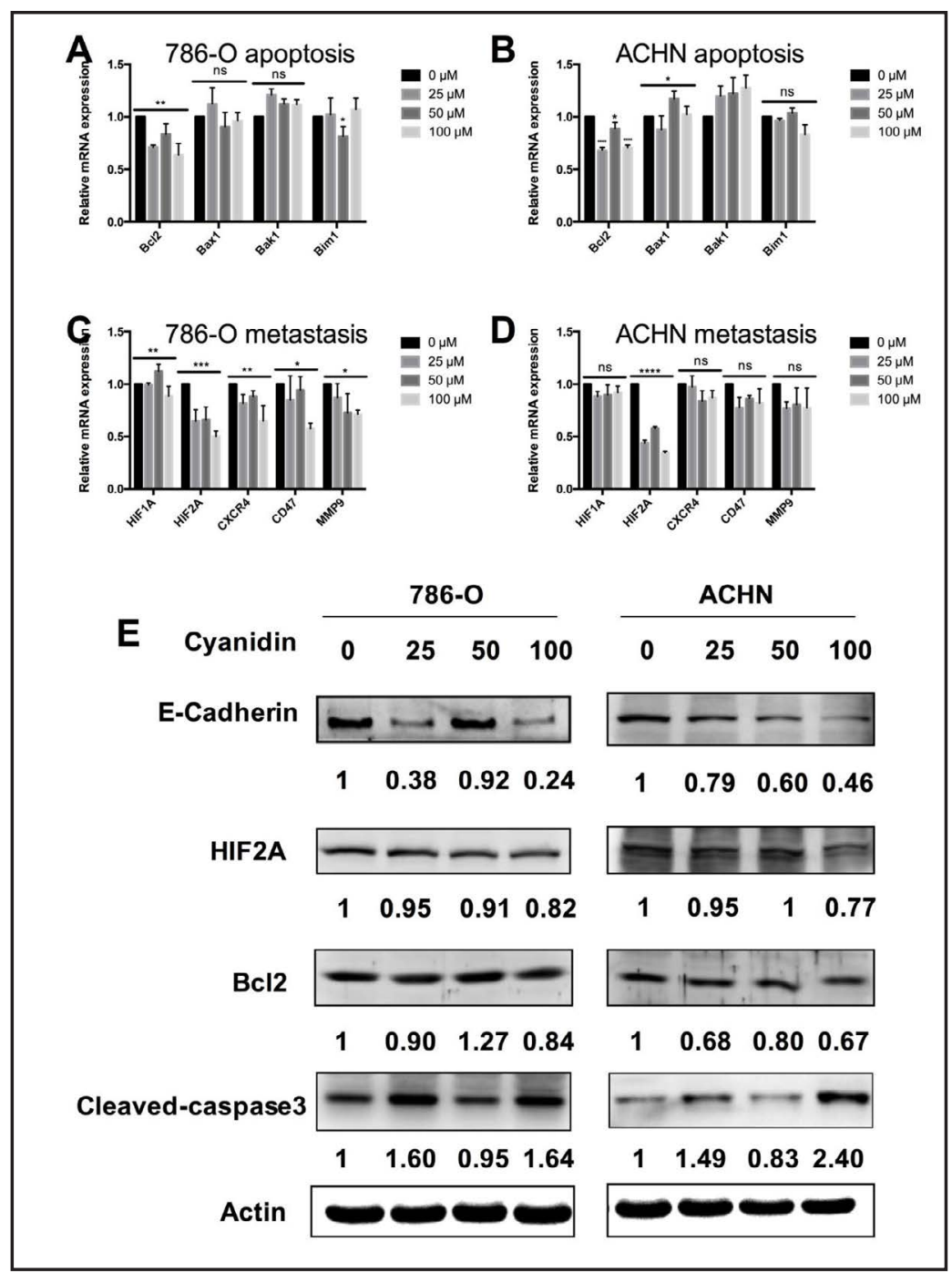

Cyanidin increases the expression of EGR1 and decreases the expression of SEPW1

In an investigation into the mechanism of cyanidin-mediated inhibition of the proliferation and migration of RCC cells, mRNA expression RNA-seq (Illumina 2000) was used to determine the genes that showed changes in their expression levels. The genes most upregulated and most downregulated in the $100 \mu \mathrm{M}$ cyanidin group were selected and compared with the control group. According to the observed fold-changes $(>2)$ and significance level $(p<0.05)$, two genes (EGR1 and SEPW1) were screened (Fig. 4 A and B). In addition, RT-PCR confirmed that EGR1 was upregulated and SEPW1 was downregulated in the 786- 0 cells treated with cyanidin $(100 \mu \mathrm{M}$; Fig. 4D-4G). Furthermore, under the special considerations that deserve the comparison of informatic data with the results presented here, we found that the expression level of EGR1 was lower and that of SEPW1 was higher in RCC tissues than normal tissues (Fig. 5H and I). Kaplan-Meier analysis revealed that the lower EGR1 mRNA expression correlated with a lower survival rate in RCC patients (Fig. 4J) while there was no statistically significant association between the expression of SEPW1 and the survival rates in RCC (Fig. 4K). Based on the RNA-seq results (Fig. 4A-C), it appears that the genes associated with cyanidin are mainly related to apoptosis and autophagy pathways. These results indicate that cyanidin activates the expression of EGR1 and inhibits the expression of SEPW1, while simultaneously inhibiting the proliferation and migration of RCC cells. 
Effect of cyanidin on mRNA and protein expression in RCC cells

The study found that cyanidin upregulated EGR1 and downregulated SEPW1. EGR1 is a type of transcription factor containing zinc finger domains, and has been shown to be associated with apoptosis, metastasis, and autophagy in tumorigenesis [27, 28]. SEPW1 is a highly conserved small thioredoxin-like protein required for cell cycle progression and apoptosis. Previous studies have shown that quercetin (a flavonoid) induces apoptosis in colon carcinoma cells via EGR1 [25]; furthermore, it has been confirmed that EGR1 and SEPW1 are required for the progression of apoptosis, while EGR1 is also required for epithelial mesenchymal transition [29]. This study, therefore, sought to determine whether the signaling pathways of apoptosis and metastasis were directly influenced by cyanidin. We confirmed through RT-PCR that the expression of Bcl2 decreases when the cells are co-cultured with cyanidin (Fig. 5A and B), and cyanidin decreases the expression of Bcl2 and cleaved caspase- 3 in western blot (Fig. 5E). These results indicated that, first, cyanidin possibly activates the apoptosis signaling pathway because both the HIF2A mRNA levels and protein expression of E-cadherin decreased after the co-culture of cells with cyanidin (Fig. $5 \mathrm{C}-\mathrm{E})$. Second, the results indicated that cyanidin may decrease the expression of proteins involved in metastasis signaling pathways.

\section{Cyanidin-reduced ROS and autophagy}

Many previous reports have shown that cyanidin influences ROS and inhibits oxidativestress-induced autophagy. This study further explored whether an increase in EGR1 induced by cyanidin would cause a decrease in ROS and whether EGR1 influences the autophagy signaling pathway. Since 786-O and ACHN exhibit different levels of sensitivity to cyanidin treatment, this study further investigated the redox state of the cells by estimating their ROS levels in both the control and cyanidin groups. The ROS levels were determined using the

Fig. 6. RCC reduced autophagic flux in RCC 786-O and ACHN cells. (A, B) Cells were treated with the indicated doses of cyanidin for $24 \mathrm{~h}$ and the levels of ROS were examined by flow cytometry. (C, D) Cells were treated with the indicated doses of cyanidin for $24 \mathrm{~h}$ and the levels of autophagy-related genes were determined by RT-PCR. (E) Cells were treated with the indicated doses of cyanidin for $24 \mathrm{~h}$ and the levels of p62 and LC3 were measured by western blot. (F, G) Cyanidin attenuates enhanced chemosensitivity during cisplatin treatment.

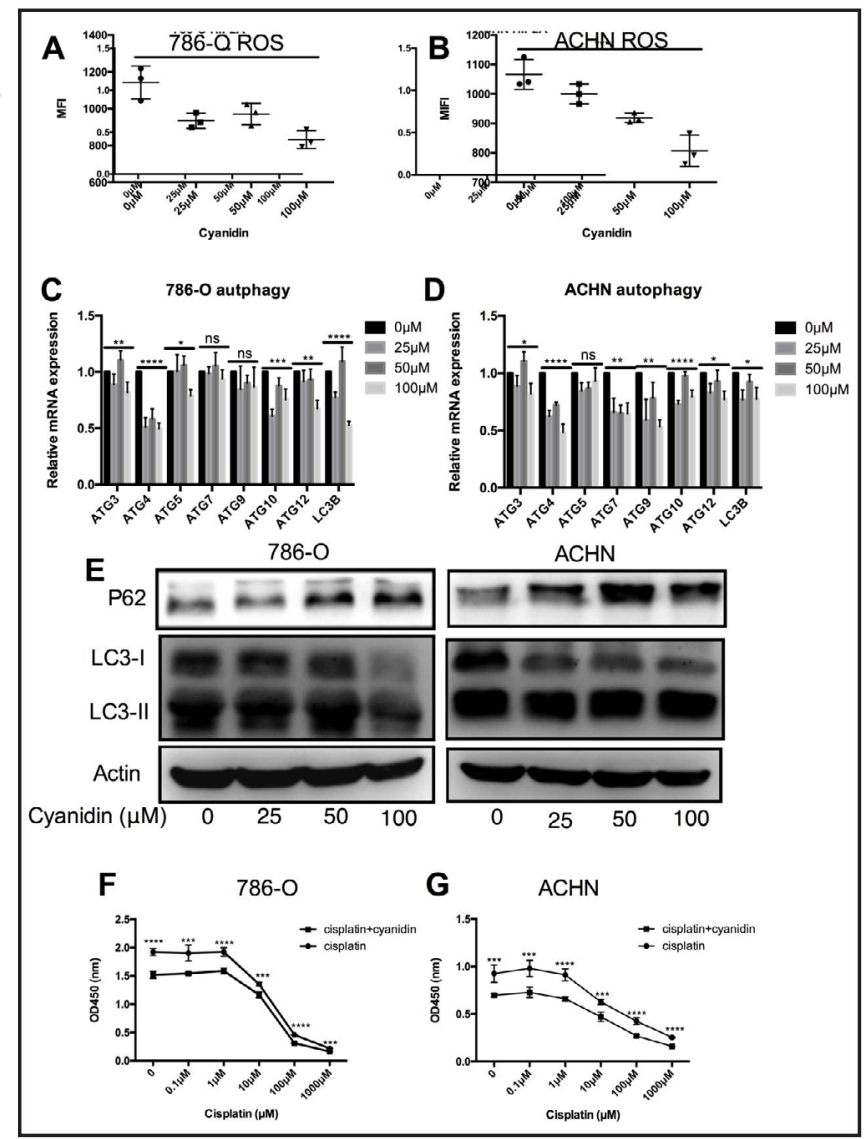


fluorescent probe DCFH-DA, in which the intensity of fluorescence is proportional to the levels of intracellular oxidant species. As shown in Fig. 6A and B, the introduction of cyanidin caused a concentration-dependent decrease of ROS production.

To further determine the effect of cyanidin on autophagy and the association of cyanidin with ROS and autophagy, genes involved in the autophagy signaling pathway were measured via qRT-PCT and western blot. It was concluded that ATG4 and p62 were downregulated while LC3-II decreased (Fig. 6C-E). Furthermore, evidence to date has shown that autophagy is rapidly activated when cancer cells are treated with chemotherapeutic drugs and plays a significant role in protecting the survival of cancer cells [30,31]. The results of this study indicate that cyanidin increases the chemosensitivity of RCC to cisplatin treatment (Fig. 7F and G).

These data suggest that the increase in EGR1 in renal cell carcinoma cells is associated with ROS levels, and EGR1 may influence autophagy signaling pathways. This supports the findings of previous studies which have suggested a relationship between EGR1 and ROS in gastric cancer [32, 33].

\section{Cyanidin reduced tumor growth in vivo}

We investigated whether cyanidin could inhibit renal cell carcinoma cells in vivo. The test results revealed that a high concentration of cyanidin $(100 \mu \mathrm{M})$ modulated RCC growth in vitro. ACHN cell suspensions $\left(1 \times 10^{6}\right)$ were injected into the right axillae of nude mice

Fig. 7. Cyanidin regulates RCC growth in vivo. (A, B) ACHN cell suspensions $\left(1 \times 10^{6}\right.$ cells $)$ were injected into the left axillae of nude mice (indicated by arrows), $100 \mu \mathrm{L}$ PBS was given twice a week to the control group and IP injections of cyanidin (6 mg/Kg in $100 \mu \mathrm{L}$ PBS) to the cyanidin group ( $\mathrm{n}$ $=5$ in each group). Tumors were harvested and visualized after 4 weeks. Images of xenograft tumors from the ACHN cells treated with cyanidin. (C) Tumors grew more slowly in the cyanidin group. (D) Tumor weights were compared between the control and cyanidin groups. (E) Histopathology of xenograft tumors. The tumor sections of the control and cyanidin groups were H\&E stained and IHC stained using antibodies against Ki-67. ${ }^{*} \mathrm{p}<0.05$, ${ }^{* *} \mathrm{p}<0.01$.

\section{KARGER}

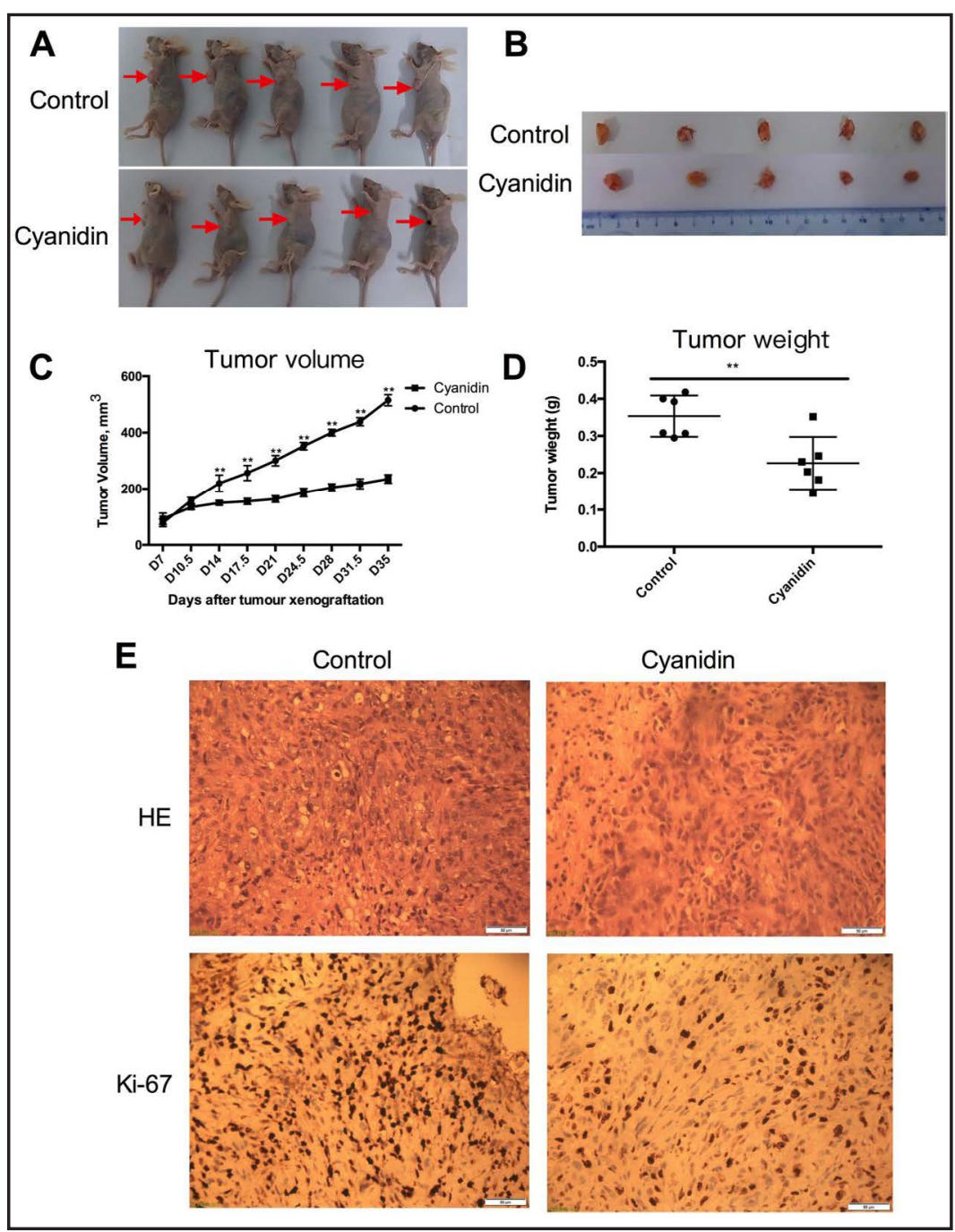


and the results revealed that a high concentration of cyanidin inhibited renal cell carcinoma growth and reduced the volumes (Fig. 7A-C) and weights of the tumors (Fig. 7D). Moreover, the expression of Ki67 was lowered (Fig. 7E). The data agree with the results of in vitro proliferation assays and suggest that a high concentration of cyanidin in cells inhibits the growth of renal cell carcinoma. Therefore, our study indicated that cyanidin suppressed the proliferation and migration potential of RCC cells.

\section{Discussion}

The availability and efficacy of chemotherapy for renal cell carcinoma is currently limited; therefore, new and effective agents for renal cell carcinoma treatment are urgently needed. Anti-cancer agents extracted from natural and organic herbs have attracted increasing attention in recent years. Cyanidin has been established as an effective anti-oxidative agent that has proven protective effects on the human cardiovascular system, nervous system, diabetes, retinal degeneration, and carcinogenesis [34-38]. Increasing evidence suggests that cyanidin inhibits tumor initiation, promotion, and progression [23, 24, 39-41]. Like other common dietary flavonoids, it is assumed that cyanidin will also have an effect on the growth and migration of RCC. One previous study showed that cyanidin has an anti-tumor effect in prostate cancer [23], while another indicated that the anti-tumor role of cyanidin is due to the suppression of cyclooxygenase-2 and attenuation of PGE2 production [40]. The effect of cyanidin on renal cell carcinoma had not been previously investigated; however, this study revealed for the first time that cyanidin exerts a direct anti-tumor effect on the growth and migration of RCC. The results indicated that low $(25 \mu \mathrm{M})$ and high concentrations (100 $\mu \mathrm{M}$ ) of cyanidin played a significant role in curtailing RCC carcinogenesis, although moderate concentrations $(50 \mu \mathrm{M})$ of cyanidin had no obvious impact. It was also discovered in this study, using RNA-seq and confirmed by RT-PCR, that cyanidin inhibited RCC carcinogenesis through EGR1 and SEPW1 concentration-independently. Furthermore, under the special considerations that required the comparison of bioinformatic data with the results presented here, we found that in RCC tissues, the expression level of EGR1 was lower and that of SEPW1 was higher than in normal tissues (Fig. 5H and I). The transcriptional factor of EGR1 is expressed in response to various extracellular signals such as growth factors, cytokines, irradiation and various kinds stressors and it has been confirmed that EGR1 plays a significant role as a tumor suppressor in breast, brain, and lung cancer and inhibits growth and transformation when overexpressed [42-44]. SEPW1, a mammalian archetype of the selenoprotein $\mathrm{W}$-like family, is an ubiquitous $9 \mathrm{kDa}$ Sec-containing protein with glutathionedependent antioxidant activity [45] that promotes tumor growth in breast cancer [26]. The silencing of SEPW1 mRNA expression causes cells to accumulate in the G0/G1 phase of the cell cycle via P53 and p21 [26]. This study found that cyanidin curtails RCC carcinogenesis by targeting EGR1 and SEPW1.

This study mainly focused on three important signaling pathways during carcinogenesis, namely, apoptosis, autophagy, and metastasis. The results convincingly showed that both low and high concentrations of cyanidin have inhibitory effects on these three pathways during RCC carcinogenesis. They were also found to enhance cleavage of the apoptotic mediator caspase- 3 and enhance the inhibition of E-cadherin activation. Further exploration revealed regulation of ATG4, which is associated with autophagy in RCC. In addition, a significant number of reports have shown that cyanidin influences the biological system through redox reactions. Cancer cells often have high levels of intrinsic oxidative stress due to increased formation of ROS, which are known to be associated with many diseases through the activation of different signaling-regulated transcriptional pathways. ROS are also associated with tumor initiation, promotion, and progression [46]. The results of this study indicated that cyanidin decreased the intracellular ROS levels. Recent studies have also demonstrated that ROS are involved in the induction of autophagy in some cell types [47], while others have suggested that antioxidants may have the ability to inhibit autophagic cell death by suppressing the generation of intracellular ROS $[48,49]$. Our findings that showed 
increased expression of autophagy markers in the $\mathrm{H}_{2} \mathrm{O}_{2}$ group indicated that the increase in cell death is related to autophagy. The results further demonstrated that oxidativestress-induced autophagic cell death in RCC cells can be reduced by cyanidin. Additionally, accumulating evidence suggests that autophagy is rapidly activated when cancer cells are treated with chemotherapeutic drugs, which is a phenomenon that plays a significant role in cancer survival $[30,31]$. Furthermore, our results indicated that cyanidin increases the chemosensitivity during cisplatin treatment.

There are, however, certain limitations to this study. The mechanism by which cyanidin increases EGR1 expression and decreases SEPW1 expression in RCC cells remains unknown, and further investigations are required to explore the potential underlying mechanisms. In addition, it is also possible that EGR1 and SEPW1 represent other targets involved in this therapy. An in-depth examination may contribute to a full understanding of their roles in the control of RCC cell invasion.

\section{Conclusion}

In summary, we found that cyanidin inhibits the proliferation and migration of RCC cells in a concentration-independent manner. Cyanidin exerts this function by increasing the expression of EGR1 and decreasing that of SEPW1. The data revealed the previously unappreciated roles of EGR1 and SEPW1 in the regulation of cyanidin-mediated cell invasion and migration, and it is concluded that cyanidin may have therapeutic potential to curtail the carcinogenic process and attenuate the chemosensitivity of RCC.

The dose-independent effect of cyanidin on carcinogenesis provides new insight into the consumption of this compound in daily life. It is worth investigating the relationship between the optimum intake of cyanidin and the carcinogenesis of RCC because different doses may exhibit different protective effects.

\section{Acknowledgements}

This work is supported by National Natural Science Foundation of China (Nos. 81170705 and 81230017). We thank Dr. Qingjian Wu for the constructive comments and for editing the manuscript. We are grateful to the authors and publications not cited in this review due to space restrictions.

LL, JX, and ZY conceived and designed the experiments; XL, DZ, YH, and QL performed the experiments; LL, YH, YW, XL, and XL analyzed the data; LL and QW contributed reagents/ materials/analysis tools; and LL and XL wrote the paper.

\section{Disclosure Statement}

The authors have no competing interests to declare.

\section{References}

1 McDermott DF: Immunotherapy of metastatic renal cell carcinoma. Cancer 2009;115:2298-2305.

-2 Escudier B, Eisen T, Stadler WM, Szczylik C, Oudard S, Siebels M, Negrier S, Chevreau C, Solska E, Desai AA, Rolland F, Demkow T, Hutson TE, Gore M, Freeman S, Schwartz B, Shan M, Simantov R, Bukowski RM: Sorafenib in advanced clear-cell renal-cell carcinoma. N Engl J Med 2007;356:125-134.

-3 Motzer RJ, Hutson TE, Tomczak P, Michaelson MD, Bukowski RM, Rixe O, Oudard S, Negrier S, Szczylik C, Kim ST, Chen I, Bycott PW, Baum CM, Figlin RA: Sunitinib versus interferon alfa in metastatic renal-cell carcinoma. N Engl J Med 2007;356:115-124.

4 Efferth T, Li PC, Konkimalla VS, Kaina B: From traditional Chinese medicine to rational cancer therapy. Trends Mol Med 2007;13:353-361. 


\section{Cellular Physiology Cell Physiol Biochem 2018;46:2517-2531 \begin{tabular}{l|l} 
and Biochemistry Published online: May II, 2018 & $\begin{array}{l}\text { C } 2018 \text { The Author(s). Published by S. Karger AG, Basel } \\
\text { www.karger.com/cpb }\end{array}$ \\
\hline
\end{tabular}}

Liu et al.: Cyanidin Curtails RCC Tumorigenesis

5 Wang D, Lu Y, Zhang T, Wang K, Rinoshika A: Effect of stiffness of rolling joints on the dynamic characteristic of ball screw feed systems in a Milling Machine. Shock and Vibration 2015;2015:1-11.

6 Graf BA, Milbury PE, Blumberg JB: Flavonols, flavones, flavanones, and human health: epidemiological evidence. J Med Food 2005;8:281-290.

7 Botta B, Vitali A, Menendez P, Misiti D, Delle Monache G: Prenylated flavonoids: pharmacology and biotechnology. Curr Med Chem 2005;12:717-739.

8 Bosetti C, Rossi M, McLaughlin JK, Negri E, Talamini R, Lagiou P, Montella M, Ramazzotti V, Franceschi S, LaVecchia C: Flavonoids and the risk of renal cell carcinoma. Cancer Epidemiol Biomarkers Prev 2007;16:98-101.

-9 Grosso G, Godos J, Lamuela-Raventos R, Ray S, Micek A, Pajak A, Sciacca S, D’Orazio N, Del Rio D, Galvano F: A comprehensive meta-analysis on dietary flavonoid and lignan intake and cancer risk: Level of evidence and limitations. Mol Nutr Food Res 2017;61

10 Batra P, Sharma AK: Anti-cancer potential of flavonoids: recent trends and future perspectives. 3 Biotech 2013;3:439-459.

11 Lim W, Jeong W, Song G: Delphinidin suppresses proliferation and migration of human ovarian clear cell carcinoma cells through blocking AKT and ERK1/2 MAPK signaling pathways. Mol Cell Endocrinol 2016;422:172-181.

12 Cao J, Wang H, Chen F, Fang J, Xu A, Xi W, Zhang S, Wu G, Wang Z: Galangin inhibits cell invasion by suppressing the epithelial-mesenchymal transition and inducing apoptosis in renal cell carcinoma. Mol Med Rep 2016;13:4238-4244.

13 Woo SM, Min KJ, Chae IG, Chun KS, Kwon TK: Silymarin suppresses the PGE2 -induced cell migration through inhibition of EP2 activation; G protein-dependent PKA-CREB and G protein-independent SrcSTAT3 signal pathways. Mol Carcinog 2015;54:216-228.

14 Ross JA, Kasum CM: Dietary flavonoids: bioavailability, metabolic effects, and safety. Annu Rev Nutr 2002;22:19-34.

15 Schippers PG, Prop FJ: Delphinidin- and cyanidin-chlorides, promotors of cell aggregation in vitro. Cell Biol Int Rep 1978;2:231-235.

16 Chen G, Bower KA, Xu M, Ding M, Shi X, Ke ZJ, Luo J: Cyanidin-3-glucoside reverses ethanol-induced inhibition of neurite outgrowth: role of glycogen synthase kinase 3 Beta. Neurotox Res 2009;15:321-331.

17 Dreiseitel A, Korte G, Schreier P, Oehme A, Locher S, Domani M, Hajak G, Sand PG: Berry anthocyanins and their aglycons inhibit monoamine oxidases A and B. Pharmacol Res 2009;59:306-311.

-18 Olivas-Aguirre FJ, Rodrigo-Garcia J, Martinez-Ruiz ND, Cardenas-Robles AI, Mendoza-Diaz SO, AlvarezParrilla E, Gonzalez-Aguilar GA, de la Rosa LA, Ramos-Jimenez A, Wall-Medrano A: Cyanidin-3-0-glucoside: Physical-Chemistry, Foodomics and Health Effects. Molecules 2016;21

19 Marczylo TH, Cooke D, Brown K, Steward WP, Gescher AJ: Pharmacokinetics and metabolism of the putative cancer chemopreventive agent cyanidin-3-glucoside in mice. Cancer Chemother Pharmacol 2009;64:12611268.

20 He J, Giusti MM: Anthocyanins: natural colorants with health-promoting properties. Annu Rev Food Sci Technol 2010;1:163-187.

-21 Paixao J, Dinis TC, Almeida LM: Dietary anthocyanins protect endothelial cells against peroxynitriteinduced mitochondrial apoptosis pathway and Bax nuclear translocation: an in vitro approach. Apoptosis 2011;16:976-989.

22 Li X, Xu J, Tang X, Liu Y, Yu X, Wang Z, Liu W: Anthocyanins inhibit trastuzumab-resistant breast cancer in vitro and in vivo. Mol Med Rep 2016;13:4007-4013.

-23 Sorrenti V, Vanella L, Acquaviva R, Cardile V, Giofre S, Di Giacomo C: Cyanidin induces apoptosis and differentiation in prostate cancer cells. Int J Oncol 2015;47:1303-1310.

24 Liu W, Xu J, Liu Y, Yu X, Tang X, Wang Z, Li X: Anthocyanins potentiate the activity of trastuzumab in human epidermal growth factor receptor 2-positive breast cancer cells in vitro and in vivo. Mol Med Rep 2014;10:1921-1926.

25 Lim JH, Park JW, Min DS, Chang JS, Lee YH, Park YB, Choi KS, Kwon TK: NAG-1 up-regulation mediated by EGR-1 and p53 is critical for quercetin-induced apoptosis in HCT116 colon carcinoma cells. Apoptosis 2007;12:411-421.

-26 Hawkes WC, Alkan Z: Delayed cell cycle progression from SEPW1 depletion is p53-and p21-dependent in MCF-7 breast cancer cells. Biochem Biophys Res Commun 2011;413:36-40.

27 Jeung YJ, Kim HG, Ahn J, Lee HJ, Lee SB, Won M, Jung CR, Im JY, Kim BK, Park SK, Son MJ, Chung KS: Shikonin induces apoptosis of lung cancer cells via activation of foxo3a/egr1/sirt1 signaling antagonized by p300 


\section{Cellular Physiology Cell Physiol Biochem 2018;46:2517-2531 \begin{tabular}{l|l} 
and Biochemistry Published onlIne: VlVay II, 2018 & $\begin{array}{l}\text { (c) } 2018 \text { The Author(s). Published by S. Karger AG, Basel } \\
\text { www.karger.com/cpb }\end{array}$
\end{tabular}}

Biochim Biophys Acta 2016;1863:2584-2593.

28 Kim J, Kang SM, Lee HJ, Choi SY, Hong SH: Oxytocin inhibits head and neck squamous cell carcinoma cell migration by early growth response-1 upregulation. Anti-cancer Drugs 2017;28:613-622.

29 Yan L, Wang Y, Liang J, Liu Z, Sun X, Cai K: Mir-301b promotes the proliferation, mobility, and epithelial-tomesenchymal transition of bladder cancer cells by targeting egr1. Biochem Cell Biol 2017;95:571-577.

-30 Zhang R, Wang R, Chen Q, Chang H: Inhibition of autophagy using 3-methyladenine increases cisplatininduced apoptosis by increasing endoplasmic reticulum stress in U251 human glioma cells. Mol Med Rep 2015;12:1727-1732.

-31 Zheng B, Zhu H, Gu D, Pan X, Qian L, Xue B, Yang D, Zhou J, Shan Y: MiRNA-30a-mediated autophagy inhibition sensitizes renal cell carcinoma cells to sorafenib. Biochem Biophys Res Commun 2015;459:234239.

-32 Ko H, Kim JM, Kim SJ, Shim SH, Ha CH, Chang HI: Induction of apoptosis by genipin inhibits cell proliferation in AGS human gastric cancer cells via Egr1/p21 signaling pathway. Bioorg Med Chem Lett 2015;25:4191-4196.

-33 Kim SJ, Kim JM, Shim SH, Chang HI: Shikonin induces cell cycle arrest in human gastric cancer (AGS) by early growth response 1 (Egr1)-mediated p21 gene expression. J Ethnopharmacol 2014;151:1064-1071.

34 Khoo HE, Azlan A, Nurulhuda MH, Ismail A, Abas F, Hamid M, Roowi S: Antioxidative and cardioprotective properties of anthocyanins from defatted dabai extracts. Evid Based Complement Alternat Med 2013;2013:434057.

-35 Wong DY, Musgrave IF, Harvey BS, Smid SD: Acai (Euterpe oleraceae Mart.) berry extract exerts neuroprotective effects against beta-amyloid exposure in vitro. Neurosci Lett 2013;556:221-226.

-36 Yao Y, Yang X, Tian J, Liu C, Cheng X, Ren G: Antioxidant and antidiabetic activities of black mung bean (Vigna radiata L.). J Agric Food Chem 2013;61:8104-8109.

-37 Lee SH, Jeong E, Paik SS, Jeon JH, Jung SW, Kim HB, Kim M, Chun MH, Kim IB: Cyanidin-3-glucoside extracted from mulberry fruit can reduce N-methyl-N-nitrosourea-induced retinal degeneration in rats. Curr Eye Res 2014;39:79-87.

-38 Xu M, Bower KA, Wang S, Frank JA, Chen G, Ding M, Wang S, Shi X, Ke Z, Luo J: Cyanidin-3-glucoside inhibits ethanol-induced invasion of breast cancer cells overexpressing ErbB2. Mol Cancer 2010;9:285.

39 Shin DY, Ryu CH, Lee WS, Kim DC, Kim SH, Hah YS, Lee SJ, Shin SC, Kang HS, Choi YH: Induction of apoptosis and inhibition of invasion in human hepatoma cells by anthocyanins from meoru. Ann N Y Acad Sci 2009;1171:137-148.

-40 Munoz-Espada AC, Watkins BA: Cyanidin attenuates PGE2 production and cyclooxygenase-2 expression in LNCaP human prostate cancer cells. J Nutr Biochem 2006;17:589-596.

41 Lamy S, Lafleur R, Bedard V, Moghrabi A, Barrette S, Gingras D, Beliveau R: Anthocyanidins inhibit migration of glioblastoma cells: structure-activity relationship and involvement of the plasminolytic system. J Cell Biochem 2007;100:100-111.

42 Yang M, Teng W, Qu Y, Wang H, Yuan Q: Sulforaphene inhibits triple negative breast cancer through activating tumor suppressor Egr1 Breast Cancer Res Treat 2016;158:277-286.

-43 Sakakini N, Turchi L, Bergon A, Holota H, Rekima S, Lopez F, Paquis P, Almairac F, Fontaine D, Baeza-Kallee N, Van Obberghen-Schilling E, Junier MP, Chneiweiss H, Figarella-Branger D, Burel-Vandenbos F, Imbert J, Virolle T: A Positive Feed-forward Loop Associating EGR1 and PDGFA Promotes Proliferation and Selfrenewal in Glioblastoma Stem Cells. J Biol Chem 2016;291:10684-10699.

44 Sun M, Nie FQ Zang C, Wang Y, Hou J, Wei C, Li W, He X, Lu KH: The Pseudogene DUXAP8 Promotes Nonsmall-cell Lung Cancer Cell Proliferation and Invasion by Epigenetically Silencing EGR1 and RHOB. Mol Ther 2017;25:739-751.

-45 Jeong D, Kim TS, Chung YW, Lee BJ, Kim IY: Selenoprotein W is a glutathione-dependent antioxidant in vivo. FEBS Lett 2002;517:225-228.

46 Panayiotidis M: Reactive oxygen species (ROS) in multistage carcinogenesis. Cancer Lett 2008;266:3-5.

47 Chen Y, Gibson SB: Is mitochondrial generation of reactive oxygen species a trigger for autophagy? Autophagy 2008;4:246-248.

48 Underwood BR, Imarisio S, Fleming A, Rose C, Krishna G, Heard P, Quick M, Korolchuk VI, Renna M, Sarkar S, Garcia-Arencibia M, O’Kane CJ, Murphy MP, Rubinsztein DC: Antioxidants can inhibit basal autophagy and enhance neurodegeneration in models of polyglutamine disease. Hum Mol Genet 2010;19:3413-3429.

49 Trejo-Solis C, Jimenez-Farfan D, Rodriguez-Enriquez S, Fernandez-Valverde F, Cruz-Salgado A, Ruiz-Azuara L, Sotelo J: Copper compound induces autophagy and apoptosis of glioma cells by reactive oxygen species and JNK activation. BMC Cancer 2012;12:156. 